\title{
Clinical utility of circulating pneumoproteins as diagnostic biomarkers in COVID-19: a systematic review and meta-analysis
}

Yani Ke

Yuqing Zhu

Jie $\mathrm{Hu}$

Shuaihang Chen

Shan Liu ( $\sim$ graystar92@163.com )

Systematic Review

Keywords:

Posted Date: January 31st, 2022

DOI: https://doi.org/10.21203/rs.3.rs-1311292/v1

License: (c) (1) This work is licensed under a Creative Commons Attribution 4.0 International License.

Read Full License 


\section{Abstract}

This study explored circulating pneumoproteins in the diagnosis, severity, and prognosis of COVID-19 by meta-analysis. We searched six English databases until December 16, 2021. Standardised mean difference (SMD) and 95\% confidence interval (Cl) were the overall outcomes. RevMan 5.3, Stata 16, and Meta-DiSc 1.4 were utilised for pooled analyses. Totally, 2902 subjects from 29 studies were included. COVID-19 patients had higher circulating KL-6, SP-D, and SP-A levels (SMD=1.34, 95\% Cl [0.60, 2.08]; $S M D=1.74,95 \% \mathrm{Cl}[0.64,2.84] ; S M D=3.42,95 \% \mathrm{Cl}[1.31,5.53]$, respectively) than healthy individuals. Circulating KL-6 levels were lower in survivors than in non-survivors (SMD $=-1.09,95 \% \mathrm{Cl}[-1.63,-0.55]$ ). Circulating KL-6, SP-D, and RAGEs levels in mild to moderate patients were significantly lower $(\mathrm{SMD}=-0.93,95 \% \mathrm{Cl}[-1.22,-0.65] ; \mathrm{SMD}=-1.67,95 \% \mathrm{Cl}[-2.82,-0.52] ; \mathrm{SMD}=-1.17,95 \% \mathrm{Cl}[-2.06,-0.28]$, respectively) than severe patients. Subgroup analysis and meta-regression suggested that age may be responsible for the heterogeneity $(P=0.071)$ when analysing $K L-6$ in mild to moderate vs. severe patients. The meta-analysis of diagnostic accuracy including KL-6 for severity and mortality, and SP-D for severity demonstrated that they all had limited diagnostic values. Therefore, circulating pneumoproteins (KL-6, SP-D, and RAGEs) may reflect the diagnosis, severity, and prognosis of COVID-19 to some extent.

\section{Introduction}

The first ongoing coronavirus disease 2019 (COVID-19) infection was detected in Wuhan, China and subsequently spread globally in a short time [1]. The outbreak was declared a pandemic by the World Health Organization (WHO) in March 2020, and various responses to prevent infection were quickly put in place. However, this epidemic has not yet been controlled. As of December 16, 2021, more than 273 million cases and 5.33 million patient deaths have been recorded worldwide (https://coronavirus.jhu.edu/). People infected with COVID-19 can be asymptomatic [2], but they can also have fever, cough, and even severe respiratory failure (SRF) [3-4], along with uncomfortable symptoms such as sore throat, fatigue, joint pain, and loss of smell and taste [5]. Many research [6-10] have indicated that serum markers such as ferritin, C-reactive protein (CRP), and lactate dehydrogenase $(\mathrm{LDH})$ are elevated in patients with severe disease compared to those in patients with mild disease, which may help predict disease progression. Nevertheless, the best biomarkers for assessing the severity of COVID-19 have not yet been established. Therefore, it is critical to continue the search for appropriate biomarkers to assess the disease and prognosis of COVID-19 patients at this stage.

Pneumoproteins, so-called lung-specific proteins, are produced predominantly in the lungs but are present in the circulatory system [11-12]. Changes in their expression levels are considered to be markers of lung injury and inflammation and are closely related to pulmonary homeostasis [13-14]. The most common pneumoproteins are KL-6, SP-D, SP-A, RAGEs, and CC-16. Recently, an increasing number of studies have explored the relationship between pneumoproteins and common respiratory diseases. Andreeva et al. [15] studied the expression difference of SP-D and CC-16 as pneumoproteins in chronic obstructive pulmonary disease (COPD). Salazar et al. [16] also explored the role and possible mechanism of KL-6 as a pneumoprotein in interstitial lung disease. Meanwhile, Witarto et al. [17] conducted a meta-analysis of 
KL-6 to predict the severity of COVID-19, and Nederi et al. [18] performed a meta-analysis of KL-6 as a diagnostic marker of severe COVID-19. Therefore, we speculate that there is a relationship between circulating pneumoproteins and COVID-19.

This study conducted a systematic review and meta-analysis of circulating pneumoproteins (including KL-6, SP-A, SP-D, RAGEs and CC-16) to evaluate their value in the diagnosis of COVID-19 and to clarify their correlation with the severity and prognosis of COVID-19.

\section{Methods}

\section{Search strategy}

Following the PRISMA guidelines, the entire process was carried out independently by two members. The protocol of this meta-analysis was registered in PROSPERO (No. CRD42021283569) (Additional File 1). We searched the PubMed, EMBASE, Cochrane Library, clinical.gov., GreyLit.org., and Research Square databases. The search time is until December 16, 2021. We used free words and MeSH words, and the search strategy is as follows: ('COVID-19' OR 'COVID19' OR 'SARS-CoV-2' OR 'Sars-CoV-2 infection' OR '2019 nCoV' OR '2019-nCoV infection' OR 'coronavirus' OR 'coronavirus disease 2019' OR 'SARS-CoV-2' OR 'Novel coronavirus' OR 'nCoV' OR 'nCoV pneumonia' OR 'Emerging Coronavirus' OR 'new coronavirus' OR 'corona-virus') AND ('pneumoproteins' OR 'KL-6' OR 'Krebs von den Lungen-6' OR 'SP-A' OR 'surfactant protein A' OR 'Pulmonary Surfactant Associated Protein A' OR 'SP-D' OR 'surfactant protein D' OR 'Pulmonary Surfactant Associated Protein D' OR 'CC-16' OR 'club cell secretory protein 16' OR 'RAGEs' OR 'receptor for advanced glycation end products'). Language limitation was not set during the retrieval. The references cited or incorporated into the included articles were also carefully checked to prevent omission. For articles with missing data, we attempted to contact the corresponding authors by email.

\section{Study selection}

The selection of the literature was checked by two researchers independently. The third researcher decided on the disputed part according to the previously established protocol. In case of incomplete data or unclear expression, we contacted the original authors via email.

\section{Association between pneumoproteins (including KL-6, SP-A, SP-D, RAGEs, or CC-16) and COVID-19. The} inclusion criteria were as follows: 1) studies concerned with patients diagnosed with COVID-19;2) outcomes that included at least one of the target biomarkers (KL-6, SP-A, SP-D, RAGEs, or CC-16); and 3) cohort or case-control studies. In cohort studies, the high-exposure group was severe COVID-19 or nonsurvivor COVID-19 group; the low-exposure group was mild to moderate COVID-19 group or survivor COVID-19 group. In case-control studies, COVID-19 patients were the case group, and healthy people were the control group. The exclusion criteria were as follows: 1) suspected COVID-19 patients; 2) healthy group with other lung diseases, such as pneumonia, interstitial lung disease (ILD), and acute respiratory 
distress syndrome (ARDS), in case-control studies; 3 ) unclear or repeated classification of different groups in cohort studies; 4) pneumoproteins (KL-6, SP-D, SP-A, RAGEs, or CC-16) levels in the lung tissue or alveolar lavage fluid, not in the plasma or serum; 5) articles with missing important data and no reply from the corresponding author; and 6) case report, review literature, or repetitive articles.

\section{Diagnostic value of pneumoproteins (including KL-6, SP-A, SP-D, RAGEs, or CC-16) in COVID-19. The} inclusion criteria were as follows: 1) studies concerned with COVID-19 patients diagnosed by the gold standard; 2) COVID-19 patients diagnosed with pneumoproteins (KL-6, SP-D, SP-A, RAGEs, or CC-16); and 3) sufficient outcomes to analyse the sensitivity, specificity, positive likelihood ratio (PLR), and negative likelihood ratio (NLR). The exclusion criteria were as follows: 1) other diagnostic related indices, but not pneumoprotein (KL-6, SP-D, SP-A, RAGEs, or CC-16); 2) pneumoprotein (KL-6, SP-D, SP-A, RAGEs, or CC-16) levels in the lung tissue or alveolar lavage fluid, but not in the plasma or serum; 3 ) failure to obtain complete diagnostic test data in four grids and no reply from the corresponding author; 4) case report, review literature, or repetitive articles.

\section{Data extraction and quality assessment}

Two researchers completed the data extraction independently, and the disputed parts were reviewed and decided by the third researcher. For articles with incomplete data, the corresponding authors were contacted by e-mail to clarify the information. The extracted data included the first author's last name, publication date, country of origin, Newcastle-Ottawa scale (NOS) score [19], number of cases and controls, basic information of cases and controls (such as age and sex), diagnostic methods, pneumoprotein level measurement method, levels of pneumoproteins (KL-6, SP-D, SP-A, RAGEs, CC-16), CRP levels, LDH levels, and sensitivity and specificity of the receiver operating characteristic (ROC) curve in each study. The NOS score is used for the quality evaluation of the included literature, which includes assessment of selection, comparability, and exposure. We further evaluated the quality of our study using the grading of recommendation, assessment, development, and evaluation (GRADE) approach (https://gdt.gradepro.org).

\section{Statistical analysis}

RevMan 5.3, Stata 16, and Meta-DiSc 1.4 were performed to analyse and integrate the data. All studies used standardised mean difference (SMD) as the outcome. The data of normal distribution are directly in the form of mean $\pm S D$, while the data with non-normal distribution were converted to mean $\pm S D$ through the online website (https://www.math.hkbu.edu.hk/ tongt/papers/median2mean.html). The $\mathrm{I}^{2}$ test and Cochran's Q-test were used to evaluate the heterogeneity among studies. When the heterogeneity was low $\left(P>0.05, I^{2}<50 \%\right)$, the fixed-effects model was the first choice. If high heterogeneity was found $(P<0.05$, $\left.\mathrm{I}^{2}>50 \%\right)$, the random-effects model was applied [20-23]. Furthermore, we explored the sources of 
heterogeneity using subgroup analysis or meta-regression. Diagnostic meta-analysis included the results of pooled sensitivity, specificity, PLR, NLR, diagnostic odds ratio (DOR), and summary ROC (sROC) curve drawing. For the sensitivity analysis, omission of each study was performed. In addition, Egger's test [24] was used to evaluate publication bias among the included studies.

\section{Results}

\section{Study selection}

The study was completed in accordance with the PRISMA guidelines. After screening 417 articles from five English databases and other sources, 315 studies were excluded, and 29 studies were further assessed based on the eligibility criteria. The included studies represented broad geographic representations with mixed populations. Most studies were performed exclusively in hospital settings, mainly in Europe (UK, Italy, Germany, Belgium, Portugal, Russia, The Netherlands, and Denmark), North America (USA and Mexico), and Asia (China, Japan, Turkey, and Indonesia). A total of 2902 subjects from 29 studies were included, including 310 healthy people. In terms of survival, 589 survivors and 92 nonsurvivors with COVID-19 were included in this part. In addition, 1603 patients with mild to moderate COVID-19 and 503 patients with severe COVID-19 were included when analyzing based on the severity of disease. The literature screening flowchart diagram is shown in Additional File, and the characteristics of the included studies in this meta-analysis are presented in Additional File 2.

\section{Quality assessment}

By using the NOS score to evaluate the quality of the included literature, we found that the average score was 5.76 (Additional File 2), indicating that most articles adopted a reasonable methodology. Most of the articles scored 6 points, but seven articles only received 5 points due to lack of representativeness, including D'Alessandro's, Deng's, Scotto's, Xue's (2021), He's, Herr's, and Manoppo's. Additionally, the results from GRADE system are shown in Additional File 3. The certainty of evidence was very low in our study. Because all included studies were observational studies, the certainty of evidence can only start from low. Moreover, the NOS score, high heterogeneity among studies, and some uncontrollable confounding factors further affect the certainty of evidence, making it from low to very low.

\section{Meta-analysis of circulating KL-6 levels}

Association of KL-6 levels in COVID-19 patients and healthy controls. Seven sets of data from four studies were analysed, as shown in Figure 1 (A). High heterogeneity was observed among studies $\left(P<0.00001, I^{2}=93 \%\right)$; thus, a random-effects model was chosen. COVID-19 patients have significantly higher circulating KL-6 levels than healthy individuals (SMD $=1.34,95 \% \mathrm{Cl}[0.60,2.08])$. 
Association of KL-6 levels in survival and non-survival patients with COVID-19. Two studies provided data related to circulating KL-6 levels (Figure 1 (B)). Circulating KL-6 levels were significantly lower in survival patients than in non-survival patients $(\mathrm{SMD}=-1.09,95 \% \mathrm{Cl}[-1.63,-0.55])$, with low heterogeneity between studies $\left(P=0.52, I^{2}=0 \%\right)$.

\section{Association of KL-6 levels in mild to moderate patients and severe patients with COVID-19. A pooled} analysis of 15 sets of data from 13 studies is shown in Figure 1 (C). We observed high heterogeneity among studies $\left(P<0.00001, I^{2}=78 \%\right)$; therefore, the random-effects model was applied. It was found that circulating KL-6 levels in patients with mild to moderate COVID-19 were significantly lower (SMD $=-0.93$, $95 \% \mathrm{Cl}[-1.22,-0.65])$ than those in patients with severe COVID-19.

\section{Subgroup analysis}

Subgroup analysis was performed because of the high heterogeneity among the studies (Table 1). According to the data collected, we analysed data in terms of country, age, CRP-ratio, and LDH. The results show that country, age, and LDH levels may be the sources of heterogeneity. When subgroups were distinguished by country, patients with mild to moderate COVID-19 had significantly lower circulating KL-6 levels than those with severe COVID-19 in the Chinese, Italian, and Japanese subgroups (SMD $=-0.98,95 \% \mathrm{Cl}[-1.39,-0.56]$; $S M D=-1.02,95 \% \mathrm{Cl}[-1.52,-0.51]$; $\mathrm{SMD}=-1.06,95 \% \mathrm{Cl}[-1.48$, $-0.64]$, respectively). However, there was no significant difference in circulating KL-6 levels between the two in Indonesia subgroup (SMD $=0.08,95 \% \mathrm{Cl}[-0.45,0.61]$. Because there was only one article conducted in Indonesia, we cannot reasonably infer whether country is exactly the source of heterogeneity. If subgroup analysis was carried out by age, mild to moderate patients aged $<49$ years and those aged $\geq 54$ years had significantly lower circulating $K L-6$ levels than severe patients $(S M D=-1.22$, $95 \% \mathrm{Cl}[-1.51,-0.93] ; \mathrm{SMD}=-1.21,95 \% \mathrm{Cl}[-1.70,-0.73])$. No significant difference was found between the two groups with $49 \leq$ age $<54(\mathrm{SMD}=-0.63,95 \% \mathrm{Cl}[-1.38,0.11])$, with high heterogeneity $\left(\mathrm{I}^{2}=80 \%\right.$, $\mathrm{P}=0.007)$. Based on these results, we hypothesised that age might partly explain the high heterogeneity. If classified by $\mathrm{LDH}$, circulating $\mathrm{KL}-6$ levels in mild to moderate patients with $\mathrm{LDH}<300$ were significantly lower than those in severe patients (SMD $=-1.22,95 \% \mathrm{Cl}[-1.51,-0.93])$. There was no significant difference between the two groups with $\mathrm{LDH} \geq 300(\mathrm{SMD}=-0.93,95 \% \mathrm{Cl}[-1.93,0.06])$. Whether $\mathrm{LDH}$ is a source of heterogeneity requires further verification.

Table 1. Subgroup analysis of circulating KL-6 levels in mild to moderate patients and severe patients with COVID-19 


\begin{tabular}{|c|c|c|c|c|c|}
\hline Subgroup & Data sets & Model & SMD $(95 \% \mathrm{Cl})$ & $\mathrm{P}$ & $\mathrm{I}^{2}(\%)$ \\
\hline \multicolumn{6}{|l|}{ Country } \\
\hline China & 8 & 1 & $-0.98[-1.39,-0.56]$ & $<0.00001$ & 83 \\
\hline Indonesia & 1 & 1 & $0.08[-0.45,0.61]$ & 1 & 1 \\
\hline Italy & 3 & 1 & $-1.02[-1.52,-0.51]$ & 0.29 & 20 \\
\hline Japan & 3 & 1 & $-1.06[-1.48,-0.64]$ & 0.13 & 50 \\
\hline Total & 15 & Random & $-0.93[-1.22,-0.65]$ & $<0.00001$ & 78 \\
\hline \multicolumn{6}{|l|}{ age } \\
\hline Age $<49$ & 4 & 1 & $-1.22[-1.51,-0.93]$ & 0.34 & 10 \\
\hline 49 sage $<54$ & 3 & 1 & $-0.63[-1.38,0.11]$ & 0.007 & 80 \\
\hline age $\geq 54$ & 5 & 1 & $-1.21[-1.70,-0.73]$ & 0.04 & 60 \\
\hline Total & 12 & Random & $-1.06[-1.39,-0.72]$ & $<0.0001$ & 74 \\
\hline \multicolumn{6}{|l|}{ CRP-ratio } \\
\hline CRP-ratio $<0.25$ & 4 & 1 & $-1.40[-1.79,-1.02]$ & 0.12 & 49 \\
\hline CRP-ratio $\geq 0.25$ & 7 & 1 & $-0.55[-0.85,-0.26]$ & 0.06 & 50 \\
\hline Total & 11 & Random & $-0.85[-1.23,-0.48]$ & $<0.00001$ & 82 \\
\hline \multicolumn{6}{|l|}{ LDH } \\
\hline $\mathrm{LDH}<300$ & 5 & 1 & $-1.22[-1.51,-0.93]$ & 0.29 & 20 \\
\hline $\mathrm{LDH} \geq 300$ & 1 & 1 & $-0.93[-1.93,0.06]$ & 1 & 1 \\
\hline Total & 6 & Fixed & $-1.20[-1.47,-0.92]$ & 0.38 & 6 \\
\hline
\end{tabular}

\section{$\mathrm{Cl}$, confidence intervals; CRP, C-reactive protein; LDH, lactate dehydrogenase}

\section{Meta-regression}

Based on the results of the subgroup analysis above, we selected LDH and age for further metaregression analysis. Because the ages between the two groups were seriously mismatched, we used ageratio to complete this analysis. Table 2 presents the results of the meta-regression analysis. LDH was not found to be a relevant factor for high heterogeneity $(P=0.511)$. However, age-ratio may be partly responsible for the heterogeneity $(P=0.071 \otimes 0.1)$, indicating the role of age in leading to the high heterogeneity in our study. 
Table 2. Meta-regression of circulating KL-6 levels in mild to moderate patients and severe patients with COVID-19

Covariates No. of Studies Coefficient Standard error $t \quad P \quad 95 \% \mathrm{Cl}$

Univariate meta-regression analysis

\begin{tabular}{lllllll} 
LDH & 6 & 1.001 & 0.001 & 0.72 & 0.511 & {$[0.998,1.003]$} \\
\hline Age ratio & 12 & 11.642 & 14.615 & 1.96 & 0.079 & {$[0.710,190.910]$}
\end{tabular}

$\mathrm{Cl}$, confidence intervals; LDH, lactate dehydrogenase

\section{Meta-analysis of circulating SP-D levels}

Association of SP-D levels in COVID-19 patients and healthy controls. As shown in Figure 1 (D), the random-effects model was applied to this analysis with high heterogeneity $\left(P<0.00001, I^{2}=94 \%\right)$. The pooled analysis from six sets of data out of four studies showed that circulating SP-D levels in COVID-19 patients were significantly higher $(\mathrm{SMD}=1.74,95 \% \mathrm{Cl}[0.64,2.84])$ than in healthy individuals.

Association of SP-D levels in survival and non-survival patients with COVID-19. Further studies on survival are presented in Figure $1(E)$. High heterogeneity $\left(P=0.06, l^{2}=59 \%\right)$ was detected. There was no significant difference in circulating SP-D levels between survival patients and non-survival patients $(S M D=-0.16,95 \% \mathrm{Cl}[-0.73,0.40])$ using the random-effects model.

Association of SP-D levels in mild to moderate patients and severe patients with COVID-19. Figure 1 (F) shows the circulating SP-D levels of COVID-19 patients grouped by severity. Because of the high heterogeneity $\left(P<0.00001, I^{2}=94 \%\right)$, a random-effects model was selected. In this model, the overall SMD was -1.67 (-2.82, -0.52). Mild to moderate COVID-19 patients exhibited significantly lower SP-D levels than those with severe COVID-19.

\section{Meta-analysis of circulating SP-A levels}

Association of SP-A levels in COVID-19 patients and healthy controls. A pooled analysis of four sets of data was performed, and the detailed results are shown in Figure $1(G)$. High heterogeneity $(P<0.00001$, 
$\mathrm{I}^{2}=97 \%$ ) was observed among these studies, resulting in the use of the random-effects model. Circulating SP-A levels were significantly higher in COVID-19 patients than in healthy individuals (SMD $=3.42,95 \% \mathrm{Cl}$ $[1.31,5.53])$.

Association of SP-A levels in survival and non-survival patients with COVID-19. Survival patients may have significantly lower circulating SP-A levels than non-survival patients with SMD $=-0.57[-1.07,-0.07]$. However, only one study was included; thus, the representativeness of the result was very low.

Association of SP-A levels in mild to moderate patients and severe patients with COVID-19. Two studies provided data related to circulating SP-A (Figure $1[\mathrm{H}]$ ). In view of the high heterogeneity between studies $\left(P<0.00001, I^{2}=98 \%\right)$, a random-effects model was applied. Circulating SP-A levels in patients with mild to moderate COVID-19 were not significantly different from those with severe COVID-19 (SMD =-3.93, 95\% CI $[-8.89,1.03])$.

\section{Meta-analysis of circulating RAGEs levels}

Association of RAGEs levels in COVID-19 patients and healthy controls. A pooled analysis of the four sets of data was performed, and the detailed results are presented in Figure $1(\mathrm{I})$. High heterogeneity $\left(P<0.00001, I^{2}=95 \%\right)$ was observed among these studies; thus, the random-effects model was used. No significant difference exists in circulating RAGEs levels between COVID-19 patients and healthy individuals (SMD $=0.94,95 \% \mathrm{Cl}[-0.52,2.39])$.

Association of RAGEs levels in survival and non-survival patients with COVID-19. The only study showed that there may be no significant difference in circulating RAGEs levels between the survival and nonsurvival groups $(\mathrm{SMD}=0.39,95 \% \mathrm{Cl}[-0.35,1.14])$.

Association of RAGEs levels in mild to moderate patients and severe patients with COVID-19. The results based on the severity of COVID-19 are shown in Figure $1(\mathrm{~J})$. We chose to use the random-effects model because of the high heterogeneity $\left(P<0.00001, I^{2}=91 \%\right)$. Circulating RAGEs levels in patients with mild to moderate COVID-19 were significantly lower (SMD $=-1.17,95 \% \mathrm{Cl}[-2.06,-0.28])$ than those with severe COVID-19. 


\section{Meta-analysis of circulating CC-16 levels}

Association of CC-16 levels in survival and non-survival patients with COVID-19. Only one study was included. The level of circulating CC-16 in the survival group was not significantly lower than that in the non-survival group $(\mathrm{SMD}=-0.33,95 \% \mathrm{Cl}[-0.83,0.16])$.

\section{Meta-analysis results for diagnostic value}

\section{Diagnostic value of circulating KL-6 for severity}

Seven studies were pooled for the meta-analysis of the diagnostic accuracy. The overall sensitivity was 0.67 (95\% Cl: 0.60-0.73), and the overall specificity was 0.82 (95\% Cl: 0.79-0.85) (Table 3). Meanwhile, the pooled PLR and NLR were estimated to be 3.27 (95\% Cl: $1.90-5.62)$ and 0.42 (95\% Cl: $0.32-0.55)$. The pooled DOR of the included studies was 11.20 (95\% Cl: 7.57-16.57). Significant heterogeneity was observed in many parts of the diagnostic analysis (sensitivity: $I^{2}=66.9 \%, P=0.0059$; specificity: $I^{2}=88.8 \%$, $\left.P=0.0000 ; P L R: I^{2}=87.0 \%, P=0.0000\right)$. The sROC curve showed a shoulder-arm-shaped distribution. The corresponding Spearman correlation coefficient was $0.821(P=0.023 ; a=0.5)$, suggesting that the threshold effect existed and contributed to the heterogeneity. All the results indicated that circulating KL-6 has poor accuracy in predicting the severity of COVID-19, and the threshold effect may be the main cause of high heterogeneity.

\section{Diagnostic value of circulating KL-6 for mortality}

Only two studies were included in this meta-analysis, and the results are presented in Table 3. The pooled sensitivity, specificity, PLR, and NLR were 0.476 (95\% Cl 0.257-0.702), 0.905 (95\% Cl, 0.871-0.933), 5.792 (95\% Cl 1.106-30.336), and 0.422 (95\% Cl 0.059-3.047), respectively. Additionally, the pooled DOR was estimated to be 13.235 (95\% Cl: 1.221-143.49). Because the number of included studies was limited, we did not further explore the heterogeneity and threshold effects. This indicates that circulating $\mathrm{KL}-6$ may be an indicator of poor accuracy for predicting the mortality of COVID-19.

Table 3. Meta-analysis results for diagnostic value 


\begin{tabular}{|llllll|}
\hline Objective & Se $(95 \% \mathrm{Cl})$ & $\mathrm{Sp}(95 \% \mathrm{Cl})$ & $\mathrm{PLR}(95 \% \mathrm{Cl})$ & $\mathrm{NLR}(95 \% \mathrm{Cl})$ & $\mathrm{DOR}(95 \% \mathrm{Cl})$ \\
\hline $\begin{array}{l}\text { KL-6 for } \\
\text { severity }\end{array}$ & 0.67 & 0.82 & 3.27 & 0.42 & 11.20 \\
& $(0.60-0.73)$ & $(0.79-0.85)$ & $(1.90-5.62)$ & $(0.32-0.55)$ & $(7.57-16.57)$ \\
\hline $\begin{array}{l}\text { KL-6 for } \\
\text { mortality }\end{array}$ & 0.476 & 0.905 & 5.792 & 0.422 & 13.235 \\
& $(0.257-$ & $(0.871-$ & $(1.106-$ & $(0.059-$ & $(1.221-$ \\
& $0.702)$ & $0.933)$ & $30.336)$ & $3.047)$ & $143.49)$ \\
$\begin{array}{l}\text { SP-D for } \\
\text { severity }\end{array}$ & 0.810 & 0.859 & 5.773 & 0.244 & 24.821 \\
& $(0.581-$ & $(0.750-$ & $(3.044-$ & $(0.102-$ & $(6.582-$ \\
& $0.946)$ & $0.934)$ & $10.948)$ & $0.584)$ & $93.600)$ \\
\hline
\end{tabular}

Se, sensitivity; Sp, specificity; PLR, pooled positive likelihood ratio; NLR, negative likelihood ratio; DOR, diagnostic odds ratio

\section{Diagnostic value of circulating SP-D for severity}

Table 3 shows the meta-analysis results for the diagnostic value of circulating SP-D to identify disease severity. The pooled sensitivity and specificity of the two studies were $0.810(95 \% \mathrm{Cl}, 0.581-0.946)$ and 0.859 (95\% Cl: $0.750-0.934)$, respectively. The pooled PLR, NLR, and DOR were estimated to be 5.773 (95\% Cl: 3.044-10.948), 0.244 (95\% Cl: 0.102-0.584), and 24.821 (95\% Cl: 6.582-93.600). The results show that the pooled DOR is high, indicating that circulating SP-D may have moderate accuracy for the severity of COVID-19.

\section{Sensitivity analysis}

\section{Circulating KL-6 levels}

After each study was excluded sequentially, outcomes of circulating KL-6 levels in COVID-19 patients vs. healthy individuals and in mild to moderate vs. severe COVID-19 patients were found to be robust. In addition, because only two studies were included in the meta-analysis of survival patients vs. nonsurvival patients, no sensitivity analysis was performed.

\section{Circulating SP-D levels}

Sensitivity analysis showed that the studies excluded one by one had no significant impact on the results of the circulating SP-D levels in COVID-19 patients vs. healthy people, in survival patients vs. non-survival patients, and in mild to moderate vs. severe COVID-19 patients. 


\section{Circulating SP-A levels}

Due to the number of studies included, we only conducted a sensitivity analysis for circulating SP-A levels in COVID-19 patients vs. healthy people. By excluding one of the studies, the result was completely beyond the original range.

\section{Circulating RAGEs levels}

After excluding the studies one by one, no significant impact was made on the results of circulating SP-A levels in COVID-19 patients vs. healthy people and in mild to moderate vs. severe COVID-19 patients.

\section{Publication bias}

According to the Egger's test, the P-values were all greater than 0.05, except for the results of circulating SP-D levels in mild to moderate vs. severe COVID-19 patients and circulating SP-A levels in COVID-19 patients vs. healthy people, indicating a certain probability of publication bias. We further carried out the metatrim method for the two studies, and the results of circulating SP-D levels in mild to moderate vs. severe COVID-19 patients was relatively robust. The result of circulating SP-A levels in COVID-19 patients vs. healthy individuals was reversed, indicating the non-negligible existence of publication bias.

\section{Discussion}

The pathogen responsible for the worldwide pandemic of COVID-19 is the 2019 novel coronavirus (SARSCoV-2) [54]. It has been confirmed that SARS-CoV-2 binds to the angiotensin converting enzyme 2 (ACE2) receptor on the surface of the host cell membrane through Spike protein [55-58], invades the host cells, and eventually leads to the occurrence of the disease [59-60]. ACE2 is widely expressed in human tissues, especially in the alveolar epithelial cells [60]. The lungs are undoubtedly the primary target of SARS-CoV2. When the patient's inflammatory response continues to progress, cellular responses induce apoptosis of normal lung tissue and further damage to the alveolar structure. Studies have observed that some COVID-19 patients have pulmonary interstitial fibrosis and type II alveolar epithelial cell hyperplasia [61]. In addition, both immunohistochemical and electron microscopic findings suggest ruin of type II alveolar epithelial cells with mild to moderate hyperplasia [62].

Krebs von den Lungen-6 (KL-6) is a high molecular weight glycoprotein secreted by type II alveolar and bronchiolar epithelial cells [63-65], which promotes chemotactic activity and anti-apoptotic effects in human lung fibroblasts. Surface protein A (SP-A) and D (SP-D) are collagen glycoproteins mainly secreted by type II alveolar cells and have an innate immune defence function [66]. They can participate 
in the host's innate defence against microorganisms and regulate the adaptive immune response, both of which play an important role in maintaining lung health. Receptors for advanced glycation end-products (RAGEs) are 35-KDA proteins, which are also recognised as a member of the immunoglobulin superfamily and a hallmark of alveolar epithelial injury [67]. Meanwhile, the RAGE pathway has been proven to be involved in the pathogenesis of lung diseases such as COPD, ILD, and ARDS [68]. Clara cell secretory protein-16 (CC-16) is a $16 \mathrm{kDa}$ pneumoprotein produced predominantly by club cells [69], which can be found in respiratory bronchioles and from the non-ciliated columnar cells of the large and small airways [70].

It has been speculated that SARS-CoV-2 can induce cytopathic effects on type II lung cells, leading to lung injury. When pulmonary epithelial lesions are present, alveolar capillary leakage leads to elevated or decreased circulating pneumoproteins levels. Therefore, the expression levels of circulating pneumoproteins can reflect the condition and prognosis of COVID-19 to some degree. Thus, exploring the link between COVID-19 and pneumoproteins is of great clinical value.

First, we found 417 articles from five English databases and other sources, and only 29 of them were eventually included in the study. The included studies originated from several regions and were representative to some degree. Concurrently, we explored the relationship between circulating pneumoproteins (including KL-6, SP-A, SP-D, RAGEs, CC-16) and COVID-19 and their diagnostic values in terms of disease, disease severity, and mortality. The pooled results showed that the levels of circulating KL-6, SP-D, and SP-A in COVID-19 patients were obviously higher than those in healthy individuals, but no significant difference existed in RAGEs levels. The non-survival group had significantly lower circulating KL-6 levels than the survival group, while in terms of SP-D levels, there was no significant difference between them. Finally, circulating KL-6, SP-D, and RAGEs levels were significantly lower in patients with mild to moderate COVID-19 than those with severe COVID-19, while there was no significant difference in circulating SP-A levels between the two groups. The results of the diagnostic meta-analysis demonstrated that circulating KL-6 levels had poor accuracy in diagnosing disease severity, and there was a threshold effect leading to heterogeneity. The accuracy of KL-6 in the diagnosis of mortality and SP-D in the diagnosis of disease severity is also poor.

Most of the studies included has high heterogeneity. However, due to the limited number of studies included in some parts, we finally selected the data of circulating KL-6 in mild to moderate vs. severe patients for the subgroup analysis and meta-regression, hoping to find the sources of high heterogeneity. The subgroup analysis showed that there was no significant difference between subgroups when divided by CRP-ratio. However, if we carry out subgroup analysis in terms of country, there is no significant difference in KL- 6 levels between the mild to moderate and severe groups in the subgroup of Indonesia. When we used age as the basis for subgroup classification, there was no significant difference in KL-6 levels between mild and moderate patients with $49 \leq$ age $<54$ and severe patients. We further performed subgroup analysis according to LDH, and no significant difference was observed in KL-6 levels between mild to moderate patients with $L D H \geq 300$ and severe patients. Meta-regression was performed from the 
perspective of age and LDH. The results showed that LDH is not the source of heterogeneity, but the ageratio may act as the source of heterogeneity $(P=0.071)$.

In addition, we explored the sensitivity of each part by excluding one study at a time. Because only two studies were included in some parts, we only focused on the parts with the number of studies $\geq 3$. When we analysed SP-A levels in COVID-19 and healthy groups, the results changed with the above method. In contrast, the sensitivity analysis results of the other parts suggest that they are all robust. Publication bias was measured using a funnel plot and Egger's test. Most studies have no publication bias, except circulating SP-D levels in mild to moderate vs. severe COVID-19 patients and circulating SP-A levels in COVID-19 patients vs. healthy people. Therefore, we implemented a metatrim to determine whether the results were robust. The result of circulating SP-D levels in mild to moderate vs. severe COVID-19 patients was relatively robust, while that of circulating SP-A levels in COVID-19 patients vs. healthy people was not. Additionally, this study's certainty of evidence was evaluated to be very low according to the GRADE system.

\section{Limitations}

The present study has some limitations. First, the included studies were limited. Many studies may have some relevance, but they were excluded because the data were incomplete or the corresponding authors could not be contacted. Only 1-2 studies were included in some parts, and the pooled results may lack strong representativeness. Similarly, there are few studies that can be used to analyse diagnostic accuracy. We only analysed the diagnostic accuracy of KL-6 in disease severity and mortality, and SP-D in disease severity. Perhaps follow-up studies could focus on the accuracy of pneumoproteins in the diagnosis of COVID-19. Second, our research may have some regional bias, mainly in Europe, North America, and Asia. COVID-19 is a global disease in the last two years; thus, it is better to summarise the situations of all regions in the world to obtain more accurate conclusions. Third, there may be many confounding factors among the included studies. Due to differences in race, testing technology, and experimental operation in various countries, the values included in our study also varied greatly, even if we had converted the same indicator into the same unit. Therefore, we finally selected SMD to analyse the results. Finally, there is obviously high heterogeneity among many studies, but due to the small number of included studies, we only analysed one part (circulating KL-6 in mild to moderate vs. severe patients). Each study had different concerns, and there was little basis for subgroup classification. COVID-19 is considered as a respiratory disease affecting multiple organs of the whole body, which may be reflected in haematological indices and lung images. However, because few studies are concerned with these indicators, we could not summarise the data and analyse them from these points.

\section{Conclusions}

We explored the relationship between circulating pneumoproteins and COVID-19 in terms of disease, disease severity, and mortality. The results showed that circulating KL-6, SP-D, and SP-A levels in COVID19 patients were significantly higher than those in healthy people. The non-survival group had 
significantly higher circulating KL-6 levels than the survival group. The levels of circulating KL-6, SP-D, and RAGEs in mild to moderate patients with COVID-19 were significantly lower than those in severe patients. Furthermore, circulating KL-6 had poor diagnostic accuracy in disease severity and mortality, and SP-D was also poor in the diagnosis of disease severity. The results of subgroup analysis and metaregression suggest that age may be the source of heterogeneity when analysing circulating KL- 6 levels in mild to moderate vs. severe patients with COVID-19. As the number of included studies is limited and regional, the relationship between pneumoproteins and COVID-19 remains to be further studied. Multiregional, multi-aspect, and multi-centre studies are necessary to explore the influence of various pneumoproteins on the pathogenesis and prognosis of COVID-19 and their diagnostic values.

\section{Declarations}

\section{Acknowledgements}

We would like to appreciate for his encouragement and support.

\section{Conflict of interest}

The authors declare that they have no competing interests.

\section{References}

1. Wang C, Horby PW, Hayden FG, et al. A novel coronavirus outbreak of global health concern. Lancet 2020; 395(10223): 470-3.

2. Gao Z, Xu Y, Sun C, et al. A systematic review of asymptomatic infections with COVID-19. J Microbiol Immunol Infect 2021; 54(1): 12-6.

3. Chen N, Zhou M, Dong X, et al. Epidemiological and clinical characteristics of 99 cases of 2019 novel coronavirus pneumonia in Wuhan, China: a descriptive study. Lancet 2020; 395(10223): 507-13.

4. Lai CC, Shih TP, Ko WC, et al. Severe acute respiratory syndrome coronavirus 2 (SARS-CoV-2) and coronavirus disease-2019 (COVID-19): The epidemic and the challenges. Int J Antimicrob Agents 2020; 55(3): 105924.

5. Lechien JR, Chiesa-Estomba CM, De Siati DR, et al. Olfactory and gustatory dysfunctions as a clinical presentation of mild-to-moderate forms of the coronavirus disease (COVID-19): a multicenter European study. Eur Arch Otorhinolaryngol 2020; 277(8): 2251-61.

6. Cheng L, Li H, Li L, et al. Ferritin in the coronavirus disease 2019 (COVID-19): A systematic review and meta-analysis. J Clin Lab Anal 2020; 34(10): e23618. 
7. Vargas-Vargas M, Cortés-Rojo C. Ferritin levels and COVID-19. Rev Panam Salud Publica 2020; 44: e72.

8. Liu F, Li L, Xu M, et al. Prognostic value of interleukin-6, C-reactive protein, and procalcitonin in patients with COVID-19. J Clin Virol 2020; 127: 104370.

9. Tjendra Y, Al Mana AF, Espejo AP, et al. Predicting Disease Severity and Outcome in COVID-19 Patients: A Review of Multiple Biomarkers. Arch Pathol Lab Med 2020; 144(12): 1465-74.

10. Malik P, Patel U, Mehta D, et al. Biomarkers and outcomes of COVID-19 hospitalisations: systematic review and meta-analysis. BMJ Evid Based Med 2021; 26(3): 107-8.

11. Hermans C, Bernard A. Lung epithelium-specific proteins: characteristics and potential applications as markers. Am J Respir Crit Care Med 1999; 159(2): 646-78.

12. Jeon $D$, Chang EG, McGing $M$, et al. Pneumoproteins are associated with pulmonary function in HIV-infected persons. PLoS One 2019; 14(10): e0223263.

13. Kirkhus NE, Ulvestad B, Barregard L, et al. Pneumoproteins in Offshore Drill Floor Workers. Int J Environ Res Public Health 2019; 16(3).

14. Moon JY, Leitao Filho FS, Shahangian K, et al. Blood and sputum protein biomarkers for chronic obstructive pulmonary disease (COPD). Expert Rev Proteomics 2018; 15(11): 923-35.

15. Andreeva E, Pokhasnikova M, Lebedev A, et al. Inflammatory parameters and pulmonary biomarkers in smokers with and without chronic obstructive pulmonary disease (COPD). J Thorac Dis 2021; 13(8): 4812-29.

16. Salazar GA, Kuwana M, Wu M, et al. KL-6 But Not CCL-18 Is a Predictor of Early Progression in Systemic Sclerosis-related Interstitial Lung Disease. J Rheumatol 2018; 45(8): 1153-8.

17. Pramana Witarto A, Samarta Witarto B, Er Putra AJ, et al. Serum Krebs von den Lungen- 6 for Predicting the Severity of COVID-19 Lung Injury: A Systematic Review and Meta-Analysis. Iran Biomed J 2021; 25(6): 381-9.

18. Naderi N, Rahimzadeh M. Krebs von den Lungen-6 (KL-6) as a clinical marker for severe COVID-19: A systematic review and meta-analyses. Virology 2022; 566: 106-13.

19. Stang A. Critical evaluation of the Newcastle-Ottawa scale for the assessment of the quality of nonrandomized studies in meta-analyses. Eur J Epidemiol 2010; 25(9): 603-5.

20. Higgins JPT, Green S, Cochrane Collaboration, 2008. Cochrane Handbook for Systematic Reviews of Interventions. Cochrane Book Series. Wiley-Blackwell, Chichester, England; Hoboken, NJ. 
21. Higgins JP, Thompson SG, Deeks JJ, et al. Measuring inconsistency in meta-analyses. Bmj 2003; 327(7414): 557-60.

22. Schmidt FL, Oh IS, Hayes TL. Fixed- versus random-effects models in meta-analysis: model properties and an empirical comparison of differences in results. Br J Math Stat Psychol 2009; 62(Pt 1): 97-128.

23. Doi SA, Barendregt JJ, Khan S, et al. Advances in the meta-analysis of heterogeneous clinical trials II: The quality effects model. Contemp Clin Trials 2015; 45(Pt A): 123-9.

24. Egger M, Davey Smith G, Schneider M, et al. Bias in meta-analysis detected by a simple, graphical test. Bmj 1997; 315(7109): 629-34

25. Alay H, Laloglu E. The role of angiopoietin-2 and surfactant protein-D levels in SARS-CoV-2-related lung injury: A prospective, observational, cohort study. J Med Virol 2021; 93(10): 6008-15.

26. Awano N, Inomata M, Kuse N, et al. Serum KL-6 level is a useful biomarker for evaluating the severity of coronavirus disease 2019. Respir Investig 2020; 58(6): 440-7.

27. Bergantini L, Bargagli E, d'Alessandro M, et al. Prognostic bioindicators in severe COVID-19 patients. Cytokine 2021; 141: 155455.

28. Chen H, Qin R, Huang Z, et al. Clinical relevance of serum Krebs von den Lungen-6 levels in patients with coronavirus disease 2019. Cytokine 2021; 148: 155513.

29. d'Alessandro M, Cameli P, Refini RM, et al. Serum KL-6 concentrations as a novel biomarker of severe COVID-19. J Med Virol 2020; 92(10): 2216-20.

30. d'Alessandro M, Bergantini L, Cameli P, et al. Peripheral biomarkers' panel for severe COVID-19 patients. J Med Virol 2021; 93(3): 1230-2.

31. Deng K, Fan Q, Yang Y, et al. Prognostic roles of KL-6 in disease severity and lung injury in COVID19 patients: A longitudinal retrospective analysis. J Med Virol 2021; 93(4): 2505-12.

32. Frix AN, Schoneveld L, Ladang A, et al. Could KL-6 levels in COVID-19 help to predict lung disease? Respir Res 2020; 21(1): 309.

33. Gomes AMC, Farias GB, Dias-Silva M, et al. SARS-CoV2 pneumonia recovery is linked to expansion of innate lymphoid cells type 2 expressing CCR10. Eur J Immunol 2021; 51(12): 3194-201.

34. He L, Lu L, Zong M, et al. The significance of $K L-6$ as prognosis monitoring biomarker in patients with severe COVID-19 from stabilized stage toward convalescence. 2021.

35. Herr C, Mang S, Mozafari B, et al. Distinct Patterns of Blood Cytokines Beyond a Cytokine Storm Predict Mortality in COVID-19. J Inflamm Res 2021; 14: 4651-67. 
36. Kerget B, Kerget F, Koçak AO, et al. Are Serum Interleukin 6 and Surfactant Protein D Levels Associated with the Clinical Course of COVID-19? Lung 2020; 198(5): 777-84.

37. Kerget F, Kerget B, Iba Yilmaz S, et al. Same virus, different course: The relationship between monocyte chemoattractant protein-1 and surfactant protein-a levels and clinical course and prognosis of COVID-19. Flora 2021; 26(3): 410-8.

38. Khadzhieva MB, Gracheva AS, Ershov AV, et al. Biomarkers of air-blood barrier damage in covid-19. Obshchaya Reanimatologiya 2021;17(3): 16-31.

39. Lim A, Radujkovic A, Weigand MA, et al. Soluble receptor for advanced glycation end products (sRAGE) as a biomarker of COVID-19 disease severity and indicator of the need for mechanical ventilation, ARDS and mortality. Ann Intensive Care 2021;11(1): 50.

40. Manoppo AF, Veterini AS, Winariani. The correlation between surfactant protein-d (sp-d) serum level and intubation time on covid-19 patients in indonesia. Teikyo Medical Journal 2021; 44(4): 9951004.

41. Peng DH, Luo Y, Huang LJ, et al. Correlation of Krebs von den Lungen- 6 and fibronectin with pulmonary fibrosis in coronavirus disease 2019. Clin Chim Acta 2021; 517: 48-53.

42. Saito A, Kuronuma K, Moniwa $K$, et al. Serum surfactant protein A and D may be novel biomarkers of COVID-19 pneumonia severity 2020.

43. Scotto R, Pinchera B, Perna F, et al. Serum KL-6 Could Represent a Reliable Indicator of Unfavourable Outcome in Patients with COVID-19 Pneumonia. Int J Environ Res Public Health 2021; 18(4).

44. Shao H, Qin Z, Geng B, et al. Impaired lung regeneration after SARS-CoV-2 infection. Cell Prolif 2020; 53(12): e12927.

45. Spadaro S, Fogagnolo A, Campo G, et al. Markers of endothelial and epithelial pulmonary injury in mechanically ventilated COVID-19 ICU patients. Crit Care 2021; 25(1): 74.

46. Suryananda TD, Yudhawati R. Association of serum KL-6 levels on COVID-19 severity: A crosssectional study design with purposive sampling. Ann Med Surg (Lond) 2021; 69: 102673.

47. Tong $\mathrm{M}$, Xiong $\mathrm{Y}$, Zhu C, et al. Serum surfactant protein $\mathrm{D}$ in COVID-19 is elevated and correlated with disease severity. BMC Infect Dis 2021; 21(1): 737.

48. Wang HY, Chen LC, Zhang Y, et al. Detection of serum KL-6 and SARS-CoV-2 antibody in patients with coronavirus disease 2019 and the diagnostic value in severe disease 2021. 
49. Xue M, Zheng P, Bian X, et al. Exploration and correlation analysis of changes in Krebs von den Lungen-6 levels in COVID-19 patients with different types in China. Biosci Trends 2020; 14(4): 290-6.

50. Xue $M$, Zhang $T$, Chen $H$, et al. Krebs Von den Lungen- 6 as a predictive indicator for the risk of secondary pulmonary fibrosis and its reversibility in COVID-19 patients. Int J Biol Sci 2021; 17(6): 156573.

51. Yalcin Kehribar D, Cihangiroglu M, Sehmen E, et al. The receptor for advanced glycation end product (RAGE) pathway in COVID-19. Biomarkers 2021; 26(2): 114-8.

52. Yamaya T, Hagiwara E, Baba T, et al. Serum Krebs von den Lungen- 6 levels are associated with mortality and severity in patients with coronavirus disease 2019. Respir Investig 2021; 59(5): 596-601.

53. Fukuda $\mathrm{Y}, \mathrm{Homma} \mathrm{T}$, Inoue $\mathrm{H}$, et al. Downregulation of type III interferons in patients with severe COVID-19. J Med Virol 2021; 93(7): 4559-63.

54. Sohrabi C, Alsafi Z, O'Neill N, et al. World Health Organization declares global emergency: A review of the 2019 novel coronavirus (COVID-19). Int J Surg 2020; 76: 71-6.

55. Yao Y, Wang H, Liu Z. Expression of ACE2 in airways: Implication for COVID-19 risk and disease management in patients with chronic inflammatory respiratory diseases. Clin Exp Allergy 2020; 50(12): 1313-24.

56. Calkovska A, Kolomaznik M, Calkovsky V. Alveolar type II cells and pulmonary surfactant in COVID-19 era. Physiol Res 2021; 70(S2): S195-s208.

57. Barreda D, Santiago C, Rodríguez JR, et al. SARS-CoV-2 Spike Protein and Its Receptor Binding Domain Promote a Proinflammatory Activation Profile on Human Dendritic Cells. Cells 2021; 10(12).

58. Kumar A, Narayan RK, Prasoon P, et al. COVID-19 Mechanisms in the Human Body-What We Know So Far. Front Immunol 2021; 12: 693938.

59. Andersen KG, Rambaut A, Lipkin WI. The proximal origin of SARS-CoV-2 Nat Med. 2020; 26(4): 450-2.

60. Hoffmann M, Kleine-Weber H, Schroeder S, et al. SARS-CoV-2 Cell Entry Depends on ACE2 and TMPRSS2 and Is Blocked by a Clinically Proven Protease Inhibitor. Cell 2020; 181(2): 271-80.e8.

61. Li S, Jiang L, Li X, Lin F, Wang Y, Li B, et al. Clinical and pathological investigation of patients with severe COVID-19. JCI Insight 2020; 5(12).

62. Gerosa C, Fanni D, Cau F, et al. Immunohistochemical findings in the lungs of COVID-19 subjects: evidence of surfactant dysregulation. Eur Rev Med Pharmacol Sci 2021; 25(13): 4639-43. 
63. Ji Y, Bourke SJ, Spears M, et al. Krebs von den Lungen-6 (KL-6) is a pathophysiological biomarker of early-stage acute hypersensitivity pneumonitis among pigeon fanciers. Clin Exp Allergy 2020; 50(12): 1391-9.

64. Ko UW, Cho EJ, Oh HB, et al. Serum Krebs von den Lungen-6 level predicts disease progression in interstitial lung disease. PLoS One 2020; 15(12): e0244114.

65. Tagami Y, Hara Y, Murohashi K, et al. Comparison of Clinical Features between the High and Low Serum KL-6 Patients with Acute Exacerbation of Interstitial Lung Diseases. Can Respir J 2021; 2021: 9099802.

66. Watson A, Madsen J, Clark HW. SP-A and SP-D: Dual Functioning Immune Molecules With Antiviral and Immunomodulatory Properties. Front Immunol 2020; 11: 622598.

67. Khaket TP, Kang SC, Mukherjee TK. The Potential of Receptor for Advanced Glycation End Products (RAGE) as a Therapeutic Target for Lung Associated Diseases. Curr Drug Targets 2019; 20(6): 679-89.

68. Oczypok EA, Perkins TN, Oury TD. All the "RAGE" in lung disease: The receptor for advanced glycation endproducts (RAGE) is a major mediator of pulmonary inflammatory responses. Paediatr Respir Rev 2017; 23: 40-9.

69. Milne S, Li X, Hernandez Cordero Al, et al. Protective effect of club cell secretory protein (CC-16) on COPD risk and progression: a Mendelian randomisation study. Thorax 2020; 75(11): 934-43.

70. Dickens JA, Lomas DA. CC-16 as a biomarker in chronic obstructive pulmonary disease. Copd 2012; 9(5): 574-5.

\section{Supplementary Materials}

\section{Supplementary material 1}

\section{Prospero}

\section{Supplementary material 2}

\section{Grade}

\section{Supplementary material 3}

Table 1. Baseline characteristics of studies included in the meta-analysis

Table 2. Newcastle-Ottawa Scale (NOS) score of the included articles 
Supplementary material 4

Figure 1. Flowchart of study inclusion and exclusion

Figure 2. (A) Forest plot of sensitivity for circulating KL-6 levels for severity in COVID-19. (B) Forest plot of specificity for circulating KL-6 levels for severity in COVID-19. (C) Forest plot of positive likelihood ratio (PLR) for circulating KL-6 levels for severity in COVID-19. (D) Forest plot of negative likelihood ratio (NLR) for circulating KL-6 levels for severity in COVID-19. (E) Forest plot of diagnostic odds ratio (DOR) for circulating KL-6 levels for severity in COVID-19. (F) sROC curve of circulating KL-6 levels for severity in COVID-19.

Figure 3. (A) Sensitivity analysis of circulating KL-6 levels between COVID-19 patients and healthy group. (B) Sensitivity analysis of circulating KL-6 levels between mild to moderate patients and severe patients with COVID-19. (C) Sensitivity analysis of circulating SP-D levels between COVID-19 patients and healthy group. (D) Sensitivity analysis of circulating SP-D levels between survival patients and non-survival patients with COVID-19. (E) Sensitivity analysis of circulating SP-D levels between mild to moderate patients and severe patients with COVID-19. (F) Sensitivity analysis of circulating SP-A levels between COVID-19 patients and healthy group. (G) Sensitivity analysis of circulating RAGEs levels between COVID19 patients and healthy group. $(H)$ Sensitivity analysis of circulating RAGEs levels between mild to moderate patients and severe patients with COVID-19.

Figure 4. (A) Egger's plot of circulating KL-6 levels between COVID-19 patients and healthy group. (B) Egger's plot of circulating KL-6 levels between mild to moderate patients and severe patients with COVID19. (C) Egger's plot of circulating SP-D levels between COVID-19 patients and healthy group. (D) Egger's plot of circulating SP-D levels between survival patients and non-survival patients with COVID-19. (E) Egger's plot of circulating SP-D levels between mild to moderate patients and severe patients with COVID19. (F) Egger's plot of circulating SP-A levels between COVID-19 patients and healthy group. (G) Egger's plot of circulating RAGEs levels between COVID-19 patients and healthy group. $(\mathrm{H})$ Egger's plot of circulating RAGEs levels between mild to moderate patients and severe patients with COVID-19.

Figure 5. (A) Funnel figure of circulating KL-6 levels between COVID-19 patients and healthy group. (B) Funnel figure of circulating KL-6 levels between mild to moderate patients and severe patients with 
COVID-19. (C) Funnel figure of circulating SP-D levels between COVID-19 patients and healthy group. (D) Funnel figure of circulating SP-D levels between survival patients and non-survival patients with COVID19. (E) Funnel figure of circulating SP-D levels between mild to moderate patients and severe patients with COVID-19. (F) Funnel figure of circulating SP-A levels between COVID-19 patients and healthy group. (G) Funnel figure of circulating RAGEs levels between COVID-19 patients and healthy group. (H) Funnel figure of circulating RAGEs levels between mild to moderate patients and severe patients with COVID-19.

\section{Figures}

\section{Figure 1}

Figure 1. (A) Forest plot of circulating KL-6 levels between COVID-19 patients and healthy group. (B) Forest plot of circulating KL-6 levels between survival patients and non-survival patients with COVID-19. (C) Forest plot of circulating KL-6 levels between mild to moderate patients and severe patients with COVID-19. (D) Forest plot of circulating SP-D levels between COVID-19 patients and healthy group. (E) Forest plot of circulating SP-D levels between survival patients and non-survival patients with COVID-19. (F) Forest plot of circulating SP-D levels between mild to moderate patients and severe patients with COVID-19. (G) Forest plot of circulating SP-A levels between COVID-19 patients and healthy group. $(\mathrm{H})$ Forest plot of circulating SP-A levels between mild to moderate patients and severe patients with COVID19. (I) Forest plot of circulating RAGEs levels between COVID-19 patients and healthy group. (J) Forest plot of circulating RAGEs levels between mild to moderate patients and severe patients with COVID-19.

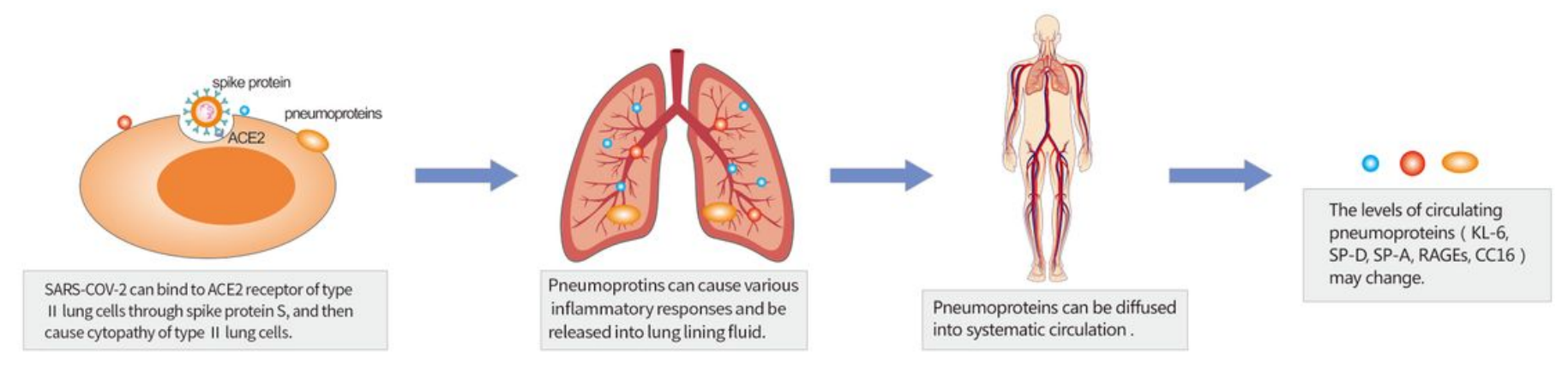

Figure 2

Figure 2. Possible mechanism of circulating pneumoproteins in COVID-19

\section{Supplementary Files}

This is a list of supplementary files associated with this preprint. Click to download. 
- Supplementarymaterial1 prospero.pdf

- Supplementarymaterial2Grade1.9.docx

- Supplementarymaterial3.docx

- Supplementarymaterial4.rar 